\title{
Spectroscopic Approaches to Tracking Metal-based Drugs in Cells and Tissue
}

\author{
Anna K. Renfrew*
}

\begin{abstract}
Metal-based drugs with novel targets and modes of action are increasingly being developed as alternatives to classical platinum(II) chemotherapeutics. Imaging methods in tumour cells and tissues offer valuable insights into the behaviour of these novel complexes; however, mapping the distribution of metal ions and complexes within cellular environments remains challenging. The advantages and limitations of three modes of imaging: synchrotron radiation-induced X-ray fluorescence, mass spectrometry, and fluorescence microscopy are discussed in this review, with particular emphasis on their use in imaging ruthenium-based drugs.
\end{abstract}

Keywords: Ruthenium · Cellular imaging · Synchrotron radiation-induced X-ray fluorescence · Mass spectrometry · Fluorescence microscopy

\section{Introduction}

Recent years have seen a marked increase in the development of metalbased drugs with mechanisms of action that are distinctly different to the classical platinum(II) complexes..1] In particular, ruthenium complexes present a diverse range of alternative properties, including inhibition of metastases, phototoxicity, and inhibition of specific enzymes (Fig. 1).[2] While the cellular targets and mechanisms of actions have been postulated or identified for many of these promising compounds, bioimaging techniques in preclinical in vitro and in vivo models are a valuable tool in the development of new metal-based drugs. ${ }^{[3]}$ An understanding of the cellular distribution of a metallodrug can aid in identifying possible targets, uptake and efflux pathways, and potential mechanisms for side effects or resistance. Not only is such information important at the cellular level, analysis of treated tumour sections can also provide vital indications on the degree to which a complex is able to penetrate and to act upon all cells within a tumour.
In spite of these incentives, the detection of metal complexes within a cellular environment is a challenging area. ${ }^{[4]}$ Total intracellular metal concentrations, or metal concentrations within a specific organelle, can be easily determined by spectroscopic techniques such as atomic absorption spectroscopy (AAS) or inductively-coupled plasma mass spectrometry (ICPMS). These approaches, however, require destruction of the cell structure, which prevents the observation of changes in the cell morphology over time and also introduces a significant risk of cross contamination and analyte loss during preparation of the samples. As such, there is much demand for spectroscopic techniques that allow direct mapping of subcellular metal distributions in intact, single cells. This review will discuss the recent advances of three such techniques, synchrotron radiation-induced X-ray fluorescence (SR-XRF), mass spectrometry, and fluorescence microscopy, with a focus on their applications to studying rutheniumbased drugs in tissues and cellular environments.

\section{Synchrotron Radiation-induced X-ray Fluorescence}

SR-XRF is increasingly being explored as a tool to study the distribution of endogenous metal ions and metal-based drugs within cells, particularly platinum complexes. ${ }^{[5]}$ This technique allows quantitative chemical analysis of an element by applying an X-ray beam to eject inner shell electrons from an atom. As outer shell electrons fill these vacancies, $\mathrm{X}$-rays are emitted corresponding to this energy difference. ${ }^{[6]}$ Elements with edge energies below that of the metal of interest can also be mapped simultaneously, allowing the distribution of endogenous elements such as phosphorus, chlorine and sulphur to be compared with the metal. This does not extend to lighter elements of biological significance such as carbon, nitrogen, and oxygen, which cannot be detected by this technique. In addition to offering spatial and quantitative information, SR-XRF can be combined with X-ray absorbance spectroscopy (XAS) to give information on the oxidation state or coordination environment of a metal. A detailed overview of SR-XRF is given in the following reviews. ${ }^{[7]}$

SR-XRF is a highly sensitive technique, with detection limits as low as $10^{-18} \mathrm{~g}$ per pixel though spatial resolution is restricted to several $\mu \mathrm{m}$, with $0.2 \mu \mathrm{m}$ being the lowest reported. As such, it is difficult to identify cellular organelles other than the nucleus and cytoplasm, however, continuous advances in synchrotron facilities and instrumentation will likely see the possibilities of resolution in the $\mathrm{nm}$ range. It should also be noted that the need for synchrotron radiation means that this technique cannot be conducted on live cells, giving only a static representation of cellular events. Furthermore, the processes required to fix cells for imaging may cause changes in elemental distribution or speciation in some cases.

To date, applications of SR-XRF to studying intracellular ruthenium distributions have been restricted to the ruthenium(III) complexes, NAMI-A and KP1019 or KP1339 (Fig. 1). Harris and coworkers used SR-XRF to study the distribution of ruthenium in single SH-SY5Y cells treated with NAMI-A or KP1019. ${ }^{[8]}$ Treatment with KP1019 led to significant ruthenium accumulation in the nuclear region, identified by its high zinc and phosphorus 

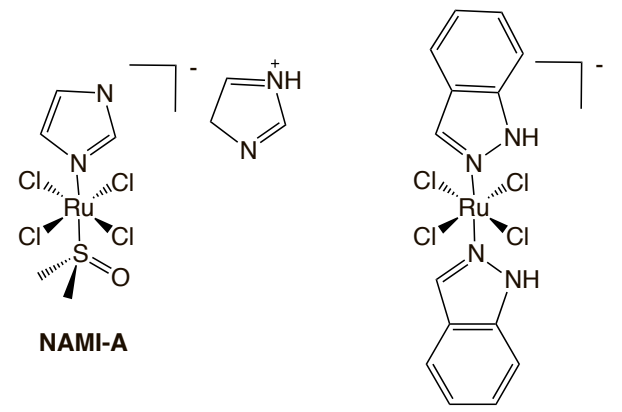
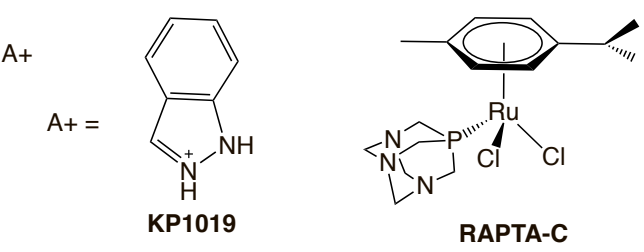

$\mathrm{Na}^{+}$

KP1339

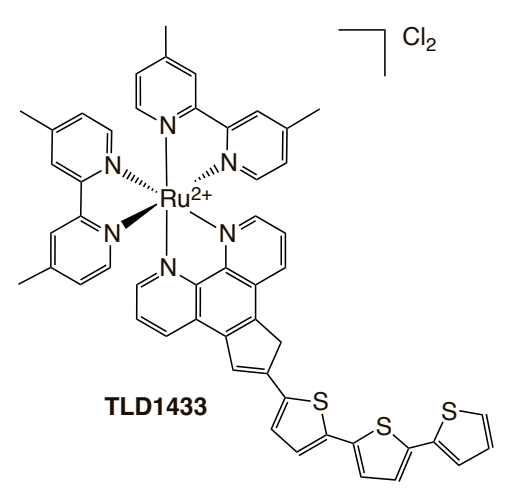

Fig. 1. Ruthenium complexes that have entered or are progressing towards clinical trials.

distribution. The percentage of ruthenium in the nucleus was quantified at $c a .50 \%$ of the total ruthenium distribution. This finding was in agreement with previous ICPMS studies that found $20-55 \%$ of the total cellular ruthenium concentration to be localised in the cell nucleus, depending on the cell line studied. The authors note that inaccuracies in determining the percentage of nuclear accumulation via SR-XRF may result from analysing a three-dimensional object projected into two dimensions. Ruthenium was also observed in the cell cytoplasm and in a region with significant iron concentration proximal to the nucleus. The colocalisation of ruthenium and iron is consistent with the suggestion that KP1019 is involved in the iron uptake and metabolism pathway. However, the authors also note that this iron pool is not present in the control cells, demonstrating that KP1019 treatment alters the cellular iron distribution, a process that could potentially contribute to the complex's mechanism of action. In contrast, treatment with NAMI-A did not alter intracellular iron distributions, nor increase intracellular ruthenium concentrations to above those of the control cells. This is in agreement with the idea that NAMI-A does not enter the cell but instead acts by interacting with extracellular or external membrane proteins.

The same authors performed a subsequent study to determine the speciation of the ruthenium complexes within treated tumour cells. ${ }^{[9]}$ To establish whether the $\mathrm{N}$-heterocyclic ligands are exchanged in the active forms of the complexes, iodinated analogues of KP1019 and NAMI-A containing 5-iodoindazole ligands were prepared. Iodine is easily detected by SR-XRF, allowing the distribution of ruthenium and the ligand to be mapped simultaneously. Analysis of single A549 cells treated with the KP1019 analogue showed significant cellular concentrations of both ruthenium and iodine, while neither element was present in the untreated control cells. The distribution of ruthenium and iodine were almost identical in the treated cells, with a low distribution throughout the cytoplasm and intense distributions in the nuclear and perinuclear regions. This suggests that both $\mathrm{Ru}-\mathrm{N}$ bonds remain intact. Importantly, incorporation of the iodine tag did not significantly alter the subcellular targeting or cytotoxicity of the KP1019 analogue. In contrast, cells treated with the iodinated analogue of NAMI-A show intracellular accumulation of ruthenium, which is not evident in treatment with NAMI-A. Treated cells had low cytoplasmic distributions of ruthenium and iodine, and highly localised regions of the two elements on and outside the cell boundaries, demonstrating again that the indazole ligand remains coordinated to ruthenium. It should be noted that NAMI-A has an imidazole not an indazole ligand therefore substitution with 5-iodoindazole significantly increases the lipophilicity of the complex and may enhance passive diffusion into the cell. It is also possible that the indazole ligand is less labile than imidazole or reduces the lability of the DMSO ligand trans to it.

SR-XRF has also been conducted on sections of SW480 tumours from mice treated with KP1339, the sodium analogue of KP1019.[10] Ruthenium was found to penetrate to all regions of the tumour tissue, with the highest intensity at the edges of the sample and near the blood vessels. As observed previously in single $\mathrm{SH}$ SY5Y cells, KP1339 treatment changed the distribution of iron with respect to the untreated control tissue sections.

\section{Laser Ablation Inductively Coupled Mass Spectrometry and Nano Secondary lonisation Mass Spectrometry}

Recent advances in mass spectrometry offer alternative mapping approaches to SR-XRF that do not require synchrotron radiation. Techniques such as MALDI (matrix-assisted laser desorption/ ionization), LA-ICPMS (laser ablation inductively coupled mass spectrometry) and Nano-SIMS (Nanoscale secondary ionisation mass spectrometry) combine the sensitivity and specificity of mass spectrometry with spatial distribution information, with the latter two approaches most applicable to studying metal ions. As with SR-XRF, analysis is conducted on fixed samples or tissue sections.

\section{Laser Ablation Inductively Coupled Mass Spectrometry}

LA-ICPMS uses high-energy focused lasers to ablate small sections of solid samples that are then analysed by ICPMS. This technique has low detection limits, in the $\mathrm{ng} / \mathrm{g}$ range but poor spatial resolution, in the $\mu \mathrm{m}$ range.[11] LA-ICPMS has the capability of measuring multiple elements simultaneously, including lighter elements such as carbon. The poor resolution of LAICPMS limits its application in mapping metal distributions in single cells, but ruthenium and other elements have been mapped in tumour tissue sections and multicellular tumour spheroids.

Egger et al. used LA-ICPMS to determine the spatial distribution of ruthenium in a series of organs from mice treated with KP1339. ${ }^{[12]}$ Ruthenium was found to be homogenously distributed in the liver and muscle, with enriched regions in the spleen. In the kidney sample, ruthenium predominately accumulated in the cortex, where platinum was also found to accumulate in mice treated with cisplatin. Interestingly, while cisplatin treatment is associated with severe nephrotoxicity, no such side effects are observed with KP1339 treatment, demonstrating that metal accumulation patterns are not necessarily a predictor of side effects.

Harris and coworkers[13] have used a similar approach to plot the distribution of ruthenium in spheroids treated with KP1019 and NAMI-A. Spheroids are three-dimensional aggregates of cultured tumour cells that have many properties to similar solid tumours, including concentration gradients of oxygen and biochemical waste products, areas of hypoxia and necrosis, and similar drug 
diffusion profiles. ${ }^{[14]}$ Both the ruthenium and carbon levels across the spheroid sections were mapped, with the carbon signal used to give an indication of cellular density throughout the sample. Strong penetration of ruthenium throughout the spheroid was observed for both complexes, demonstrating that the drugs are able to reach not only the rapidly proliferating cells on the spheroid edge but also the hypoxic and necrotic regions in the centre.

\section{Nanoscale Secondary Ion Mass Spectrometry}

Nano-SIMS uses a primary ion beam to eject low-mass secondary electrons. This technique has spatial resolution as low as $50 \mathrm{~nm}^{[15]}$ though Nano-SIMS is less sensitive than the previous techniques, with a detection limit of $0.1 \mathrm{mg} / \mathrm{g},{ }^{[11]}$ and quantification of metal concentrations is more difficult. The low ionization yield of heavier elements also makes it harder to image some metals and can necessitate very long sample irradiation times. A key advantage of Nano-SIMS is that the high mass resolution allows different isotopes to be differentiated, meaning that isotopically labelled ligands can be distinguished from endogenous elements, e.g. ${ }^{13} \mathrm{C},{ }^{15} \mathrm{~N}$. This is a powerful technique for tracking both a metal ion and its ligands within the cell environment.

Nano-SIMS has been applied to study cell samples treated with a number of platinum $^{[16]}$ and gold-based drugs, ${ }^{[17]}$ though to date only one ruthenium-treated sample has been analysed.[18] A sample of A2780CR (cisplatin resistant) cells treated with RAPTA-T was studied, where the ligands of RAPTA-T had been isotopically labelled to allow them to be distinguished from endogenous carbon and nitrogen atoms in the sample, (the toluene ligand was enriched with six ${ }^{13} \mathrm{C}$ atoms and the PTA ligand with three ${ }^{15} \mathrm{~N}$ nitrogen atoms). Ruthenium was predominantly localised on the cell membrane or interface between cells, in agreement with previous studies indicating that RAPTA-T prevents cell detachment and metastasis by interacting with cell adhesion proteins such as collagen IV and fibronectin. A clear ${ }^{15} \mathrm{~N} /{ }^{14} \mathrm{~N}$ signal representing accumulation of the PTA ligand was also visible in this region, indicating that the PTA ligand remains coordinated to ruthenium. In contrast, the ${ }^{13} \mathrm{C} /{ }^{12} \mathrm{C}$ signal is diffuse and does not indicate a clear distribution of the toluene ligand. This is attributed to partial exchange of the toluene ligand after the 24 hour incubation period and its diffusion throughout the sample. The authors acknowledge that sample preparation could also contribute to a dilution of the ${ }^{13} \mathrm{C} /{ }^{12} \mathrm{C}$ signal, however the strong ${ }^{15} \mathrm{~N} /{ }^{14} \mathrm{~N}$ signal argues against this. Small hotspots of $\mathrm{Ru},{ }^{15} \mathrm{~N}$ and, to a less- er extent, ${ }^{13} \mathrm{C}$ were also visible inside the cells, most probably in the nucleus. These are hypothesised to correspond to histone binding, as RAPTA-T is known to bind to histones.

\section{Fluorescence Microscopy}

While SR-XRF, LA-ICPMS and Nano-SIMS provide detailed information on concentration, speciation and localisation, a key disadvantage is that they cannot be performed in live cells. In this sense, fluorescence microscopy is a powerful and often complimentary technique for studying metallodrugs in live cell environments. This provides the opportunity to observe in real time and also removes risks of contamination or changes to metal distribution caused by fixing. Fluorescence microscopes have sub-micro molar resolution and can also detect three wavelengths simultaneously, meaning that the distribution of a compound of interest can be overlayed with that of two organelle stains.

An obvious limitation of fluorescence microscopy is the requirement for a compound that fluoresces within a detectable wavelength window, e.g. in the UVNIR range. In the study of metal-based drugs, a number of complexes are naturally phosphorescent, making them ideally suited for such an application. However, in the case where a complex is not luminescent, it is necessary to incorporate a fluorescent ligand or tag into the structure. Due to the bulky and lipophilic nature of most organic fluorophores, this may have a significant impact on the uptake, distribution, and toxicity of a complex, meaning that studies with such tagged complexes may not be representative of the behaviour of the original metallodrug. Furthermore, fluorescent ligands can undergo ligand exchange with the result that the fluorescence emission observed might correspond to the distribution of the ligand only and not to the tagged complex. While this may be a disadvantage, it should be noticed that fluorescence microscopy can be exploited to signal changes in coordination environment. For example, a fluorescent ligand may be quenched when coordinated to a metal, but regain its fluorescence when released, giving a turnon fluorescent signal for ligand release. Many fluorophores also have different emission wavelengths, or fluorescence lifetimes when coordinated to a metal, allowing the bound and unbound species to be distinguished spectroscopically.

\section{Luminescent Complexes}

A significant proportion of the ruthenium complexes currently under investi- gation as potential therapeutics are also luminescent. [19] This gives a convenient means of observing them in live cell imaging without the need to modify their structure. Notably, many ruthenium(II) complexes with polypyridyl ligands have a long-lived phosphorescence emission resulting from the ${ }^{3} \mathrm{MLCT}$ state. For this reason, this class of complex has also received considerable interest for use as cellular probes and sensors, often after conjugation to a specific targeting peptide. [20] Many phosphorescent complexes are also under investigation as potential cytotoxins and phototoxins. In such cases, the natural phosphorescence allows the cellular distribution of the metallodrug to be studied by confocal fluorescence microscopy. It should be noted, however, that the emission of some of these complexes is strongly dependent on environment. For example, $\left[\mathrm{Ru}(\mathrm{bpy})_{2}(\mathrm{dppz})\right]^{2+}(\mathrm{bpy}=2$,2-bipyridine, $\mathrm{dppz}=\operatorname{dipyrido}\left(3,2-\mathrm{a}: 2^{\prime}, 3^{\prime}-\mathrm{c}\right)$ phenazine $)$ is known to act as a DNA light switch, meaning that it is only emissive when intercalated to DNA. ${ }^{[21]}$ In such cases it may be necessary to confirm the fluorescence microscopy findings with another technique such as sub-cellular ICPMS. Phosphorescent ruthenium(II) polypyridyl complexes have been extensively reviewed elsewhere, ${ }^{[2,20]}$ therefore this section aims to highlight several key examples where fluorescence microscopy has helped to elucidate cellular targets rather than to provide an exhaustive summary.

Gasser et al. reported a phosphorescent complex, $\left[\mathrm{Ru}(\mathrm{dppz})_{2}(\mathrm{CppH})\right]^{2+}($ bipy $=$ 2,2'-bipyridine; $\mathrm{CppH}=2$-(2'-pyridyl) pyrimidine-4-carboxylic acid, 1, Fig. 2) that could induce apoptosis with $\mathrm{IC}_{50}$ values similar to cisplatin (Fig. 3). ${ }^{[22]}$ While the complex was capable of binding to DNA through intercalation, its mode of action did not correspond to a nuclear DNA related mechanism. Confocal fluorescence microscopy studies revealed the accumulation of the complex in the mitochondria, with very good overlay with mitotracker green FM. As complex $\mathbf{1}$ is only emissive in hydrophobic environments, the authors also conducted ICPMS on cellular fractions, confirming that $68 \%$ of the cellular ruthenium concentration was localised in the mitochondria.

$\mathrm{Xu}$ and coworkers have reported two series of phosphorescent complexes with carboline ligands that accumulate in the cytoplasm and eventually the nucleus. ${ }^{[23]}$ The authors also used TEM (transmission electron microscopy) to identify autophagic structures and vacuoles in the Ru-treated cells, identifying autophagy and apoptosis as dual modes of cell death.

Fluorescence microscopy recently was used to elucidate the target of an antibacterial dinuclear polypyridylruthenium(II) 
Fig. 2. Examples of phosphorescent ruthenium

metallodrugs 1-3.

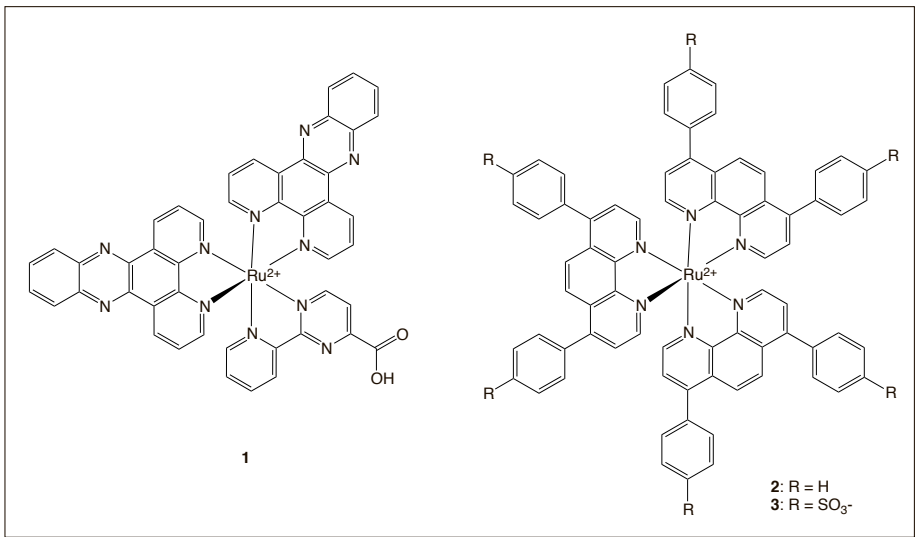

Fig. 3. Fluorescence confocal microscopy images of HeLa cells incubated with $\left[\mathrm{Ru}(\mathrm{dppz})_{2}(\mathrm{CppH})\right]^{2+}$ $(20 \mu \mathrm{M})$ for $2 \mathrm{~h}$ and Mitotracker green FM for 45 min: (a) DAPI staining; (b) cellular staining of $\left[\mathrm{Ru}(\mathrm{dppz})_{2}(\mathrm{CppH})\right]^{2+}$ (c) Mitotracker green FM staining; and (d) the overlay image. Reprinted with permission from ref. [22], J. Am. Chem. Soc. 2012, 134, 20376-20387. Copyright (2012) American Chemical Society.
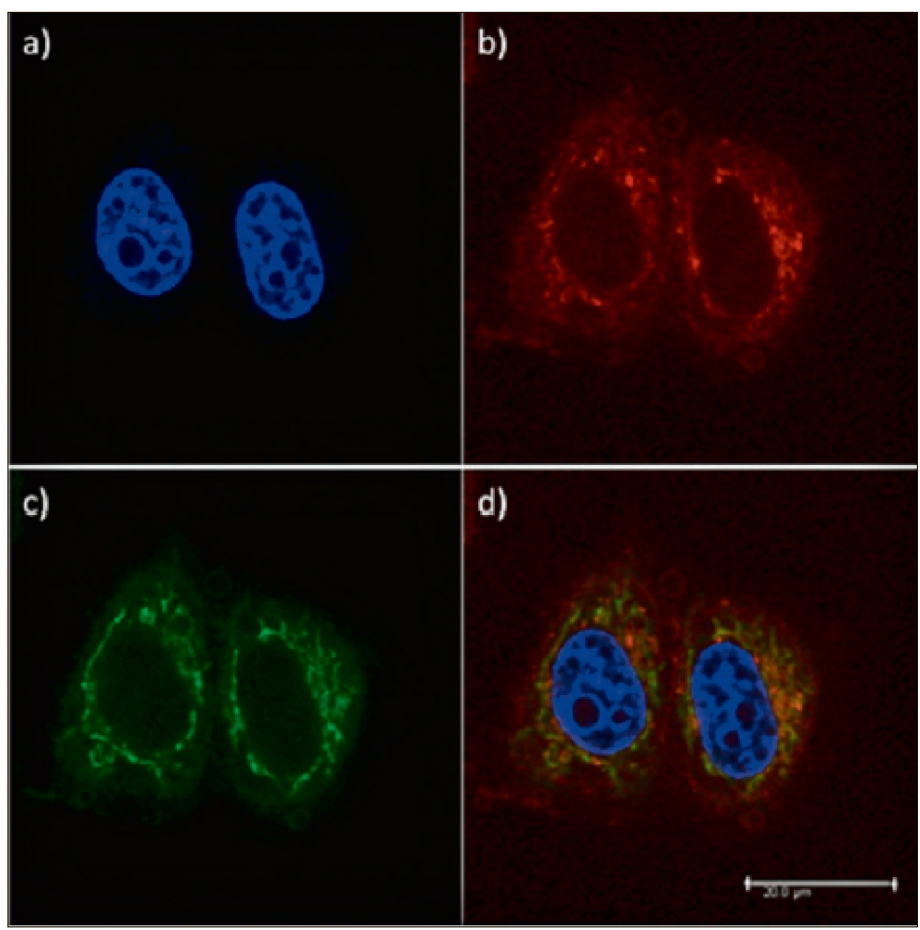

complex. The complex was found to accumulate in the ribosomes of E. Coli, selectively targeting RNA over DNA. The authors suggest that this selective targeting is responsible for the low toxicity of the complex towards mammalian cells, due to the differences in ribosome structure between bacteria and eukaryotic cells. ${ }^{[24]}$

Many phosphorescent ruthenium complexes can also act as photosensitisers, where instead of relaxing from the ${ }^{3} \mathrm{MLCT}$ state to ground state through emission, they sensitise triplet oxygen to reactive singlet oxygen. While this effect can be undesirable for some applications, ruthenium photosensitisers are gaining increasing interest for use in photodynamic therapy, with one example, TLD1433, set to enter Phase 1 clinical trials against non-invasive bladder cancer. ${ }^{[25]}$ TLD1433 (Fig. 1) has an oligothiophenefunctionalised ligand, which both enhances its phototoxicity and increases its emission. Similar to $\left[\mathrm{Ru}(\mathrm{bpy})_{2} \mathrm{dppz}\right]^{2+}$, TLD1433, displays a light-switch effect where its emission intensity is greatly enhanced on DNA binding. Confocal fluorescence microscopy studies on HL60 cells treated with TLD1433 revealed accumulation of the complex in the nucleus and nucleoli. The complex was found to be capable of causing DNA single strand breaks in combination with light, suggesting that DNA damage is the mechanism of light-induced cell death. Gasser and coworkers have also developed a series of $\left[\mathrm{Ru}(\mathrm{bpy})_{2} \mathrm{dppz}\right]^{2+}$ type complexes designed to interact with DNA.[26] Fluorescence microscopy shows accumulation of the complexes in the nucleus, and very good overlay with a DAPI nuclear stain. High resolution continuum source atom absorption spectroscopy was used to quantify the percentage of cellular ruthenium in the nucleus, revealing a greater than $50 \%$ accumulation. As for TLD1433, the complexes are capable of inducing DNA strand breaks though singlet oxygen formation, and this is proposed as a likely mode of cell death.

Glazer and coworkers demonstrated the influence the polypyridyl ligands on phototoxicity and cellular distribution by comparing two phosphorescent photosensitisers, $\quad\left[\mathrm{Ru}\left(\mathrm{Ph}_{2} \mathrm{Phen}\right)_{3}\right]^{2+}$ (2) and $\left[\mathrm{Ru}\left(\mathrm{Ph}_{2} \mathrm{Phen}-\mathrm{SO}_{3}\right)_{3}\right]^{4-}$ (3) $\left(\mathrm{Ph}_{2}\right.$ phen = bathophenanthroline; $\mathrm{Ph}_{2} \mathrm{Phen}-\mathrm{SO}_{3}=$ bathophenanthroline disulfonate) (Fig. 2).[27] Both complexes produce singlet oxygen and are significantly more toxic towards tumour cells in combination with blue light than in the dark. However, 2 is still cytotoxic in the dark $\left(\mathrm{IC}_{50}=0.6-3.75 \mu \mathrm{m}\right)$ while 3 shows no toxicity up to $300 \mu \mathrm{m}$ in any of the cell lines tested. This difference was revealed to be due to a difference in cellular accumulation. In the dark, the hydrophobic dication $\mathbf{2}$ accumulates in the mitochondria and lysosomes, while the negatively charged $\mathbf{3}$ accumulates in the cytosol. Exposure to light causes $\mathbf{3}$ to move to the lysosomes, while it does not affect the distribution of 2. Examination of the mechanism of cell death shows that $\mathbf{2}$ induces rapid mitochondrial depolarisation and follows a necrotic pathway, while $\mathbf{3}$ follows an apoptopic pathway. This study emphasizes how fine-tuning the ligands, charge and lipophilicity of a complex can strongly influence its cellular distribution, toxicity profile, and mechanism of action.

Therrien and coworkers prepared a series of $\mathrm{Ru}($ arene)pyridylporphyrin conjugates as photosensitisers, where the porphyrin moiety is responsible for the fluorescence and photosensitising properties of the complex. ${ }^{[28]}$ The pyridyl group was found to strongly affect phototoxicity, with the 4-pyridlylporphyrin around 10-fold less active than the 3-pyridyl. This difference could be explained by confocal fluorescence microscopy, which revealed that the more hydrophobic 4-pyridyl series formed aggregates inside the cytoplasm, quenching ROS production, while the 3 -pyridyl analogues were evenly distributed throughout the cytoplasm.

\section{Ruthenium Complexes with \\ Fluorescent Ligands}

In addition to phosphorescent complexes, a fluorescent ligand can also allow a ruthenium complex to be tracked by fluorescence microscopy. One difficulty in this case is that the ligand can be exchanged, meaning that any fluorescence emission observed would be due to the ligand alone and not the intact complex. In some cases, it can be easy to determine whether a fluorescent ligand is coordinated as the emission is quenched when coordinated to the metal. This can be a disadvantage if it makes the complex non-emissive but can also be an effective means of ascertaining when and where a fluorescent ligand has been released by an increase in fluorescence emission. However, obtaining quantitative information on ligand release may be difficult where it is not clear whether the emission is due to a small concentration of 
ligand or high concentration of complex, or a combination of both. Alternatively, in some cases a change in fluorescence lifetime or emission maxima can distinguish between a fluorescent ligand and its complex.

Hartinger and coworkers have prepared a series of $\mathrm{Ru}(\mathrm{II})$ arene complexes with an O,O-chelating flavonoid ligand (4) (Fig. 4). [29] The ligands are based on flavonoid natural products that are known to exhibit antioxidant, anti-inflammatory, antimicrobial and anticancer activity. Furthermore, the flavonoids are naturally fluorescent in the visible light region with an emission maximum at $520 \mathrm{~nm}\left(\lambda_{\mathrm{ex}}\right.$ $=485 \mathrm{~nm}$ ). Complexation to ruthenium does not alter the fluorescent properties of the flavonoid ligand. This allows the complexes to be tracked in live SW480 cells by confocal fluorescence microscopy. As the bidentate flavonoid ligand remains coordinated when ruthenium binds to 5'GMP, the observed emission is attributed to the ruthenium complex and not to the free flavonoid ligand. Co-localisation experiments with ER-Tracker ${ }^{\mathrm{TM}}$ Red reveal that the lead complex accumulates exclusively in the endoplasmic reticulum.

A similar approach was explored by Wang and coworkers with a Ru(II) $p$-cymene complex containing a fluorescent dppn (4,5,9,16-tetraazadibenzo[a,c]naphthacene) ligand (5, Fig. 4). ${ }^{[30]}$ The dppn ligand is highly emissive and also facilitates DNA binding. The complex $[\mathrm{Ru}(p$-cymene $)$ (dppn)(py) $]^{3+}$ shows fluorescence from a ligand-centred excited state from the dppn ligand, with an emission quantum yield of 0.31 in acetonitrile, comparable to free dppn. The emission maximum of the complex is also red-shifted $c a .60 \mathrm{~nm}$ with respect to free dppn, offering the potential to distinguish between the two species. Interestingly, the quantum yield of emission is highly dependent on the nature of the monodentate ligand: while the pyridine complex is highly emissive, the analogous chlorido complex has a quantum yield of just 0.029 , while the aqua complex is intermediate. The authors were able to

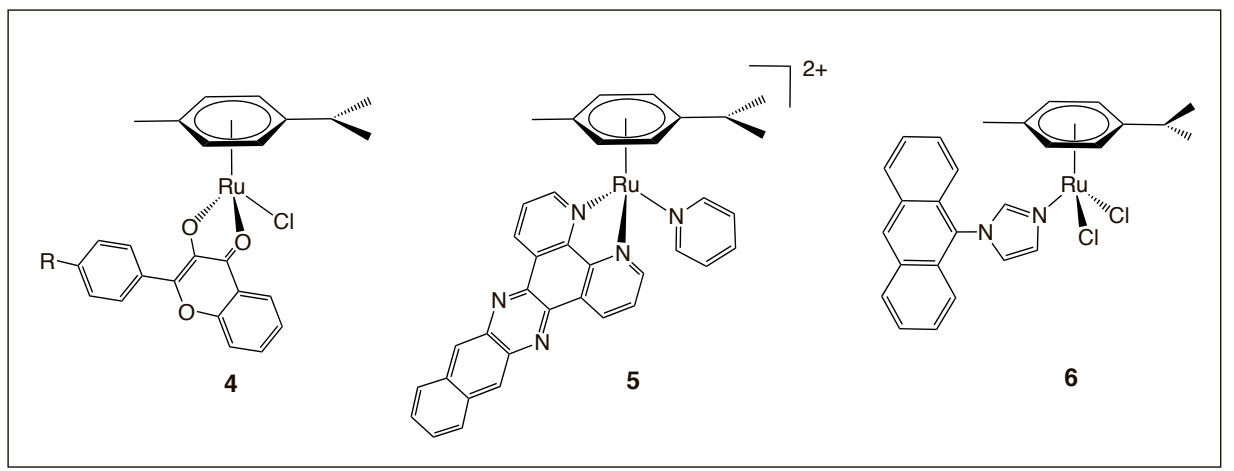

Fig. 4. Examples of ruthenium(॥)arene complexes 4-6 with fluorescent ligands. attribute this to thermal population of the LF orbital from the excited dppn LC state, leading to fluorescence quenching through non-radiative decay. The low ligand-field strength of chloride means that the LF orbital can be easily thermally populated, leading to fluorescence quenching, while the larger ligand-field strength of water and pyridine makes this process more difficult, leading to higher fluorescence quantum yields. This is an important insight when considering the design of a fluorescent tag or ligand. Subtle changes to the coordination sphere may be used to control whether the fluorescence of a ligand is quenched on coordination, which may be desirable for some applications and not for others. Confocal fluorescence microscopy in A549 cells showed localisation of both the pyridine and chloride (likely aquated) complexes in the nucleus, overlayed with a Hoechst 34580 nuclear stain. It should be noted that the emission intensity of both complexes increases seven-fold in the presence of DNA, therefore it is possible that cellular accumulation also occurs in other regions that are less visible to fluorescence microscopy.

A number of ruthenium(II)arene complexes based on the RAPTA series have been prepared with fluorescently tagged ligands to allow them to be observed by fluorescence microscopy. A complex with a fluorescent N-(anthracen-9-yl)imidazole ligand was prepared as an analogue of the PGP (P-glycoprotein) inhibitor 2-(Imidazol-1-yl)-1-(phenoxazin-10-yl)-ethanone (6, Fig. 4).[31] The fluorescence of the anthracene moiety was almost completely quenched when coordinated to ruthenium via the imidazole group, however bright emission is observed by fluorescence microscopy in the nucleus of A549 cells dosed with the complex. Cells treated with the anthracene ligand alone show a much fainter emission pattern, with no accumulation in the nucleus. This suggests that the ruthenium complex can both increase uptake and alter the cellular distribution of the ligand.
In a subsequent study, conjugation of an anthracene moiety to the arene ligand of $\mathrm{Ru}(\mathrm{II})$ arene $(\mathrm{L}) \mathrm{Cl}_{2}$ complexes $(\mathrm{L}=$ pta or sugar phosphite) was found to give a fluorescence complex, where the emission intensity of the sugar phosphite complex was around 15-fold greater than the pta complex. ${ }^{[32]}$ The spectrum of free ligand was not measured. Fluorescence microscopy of A549 cells treated with the two complexes showed faint emission in the cell membrane and cytoplasm, with no evidence of nuclear accumulation. Ru(II) arene complexes with a naphthalimide ligand tethered to either an imidazole or to the arene ligand have also been prepared but no studies were carried out in tumour cells. ${ }^{[3]}$ The fluorescence of the naphthalimide was strongly quenched on coordination to ruthenium but partly restored in the presence of DNA.

Finally, analogues of NAMI-A have been prepared by Harris and coworkers where the imidazole ligand was replaced with the fluorescent ligands 7-azaindole or an naphthalimide-conjugated imidazole. ${ }^{[34]}$ The emission of the ligands in aqueous solution was only slightly quenched on coordination to ruthenium though no studies were conducted in cells.

\section{Complexes with Fluorescence Responses to Ligand Exchange}

Sadler and coworkers developed a photolabile complex $\left[\left\{\left(\eta^{6}\right.\right.\right.$-indan $)$ $\left.\mathrm{RuCl}\}_{2}(\mu-2,3-\mathrm{dpp})\right]\left(\mathrm{PF}^{6}\right)_{2}$ where 2,3-dpp (2,3-bis(2-pyridyl)pyrazine) is a bridging ligand (7, Fig. 5).[35] Irradiation with UV light induces photosubstitution of both the chlorido and indan ligands to produce a reactive complex that can bind and crosslink plasmid DNA. The indan ligand is fluorescent but its emission is quenched when coordinated to ruthenium. Irradiation of 7 in $0.1 \mathrm{M} \mathrm{NaCl}$ solution leads to a $c a$. 40-fold increase in emission, signalling release of the indan ligand. No confocal imaging was carried out in cells, possibly due to the short excitation/emission wavelengths of indan $(260 / 295 \mathrm{~nm})$.

We have investigated complexes with the natural product curcumin as a bidentate ligand, a fluorescent compound with anticancer properties. ${ }^{[36]}$ Curcumin fluorescence was strongly quenched (100 fold reduction) when coordinated to ruthenium in the complex $\left[\mathrm{Ru}(\text { curcumin)(tpa) }]^{+}\right.$(tpa $=$ tris(2-pyridylmethyl)amine) (8, Fig. 5). Confocal fluorescence microscopy of DLD-1 cells treated with the ruthenium complex showed very weak fluorescence in the cell membrane, indicating release of curcumin in this region (Fig. 6). Cells treated with curcumin alone showed emission in the same region but at a much stronger intensity. In a 3D spheroid sample, emission from the curcumin sample was 
Fig. 5. Structures of ruthenium complexes $7-10$ that undergo a change in fluorescence emission or lifetime with ligand exchange.

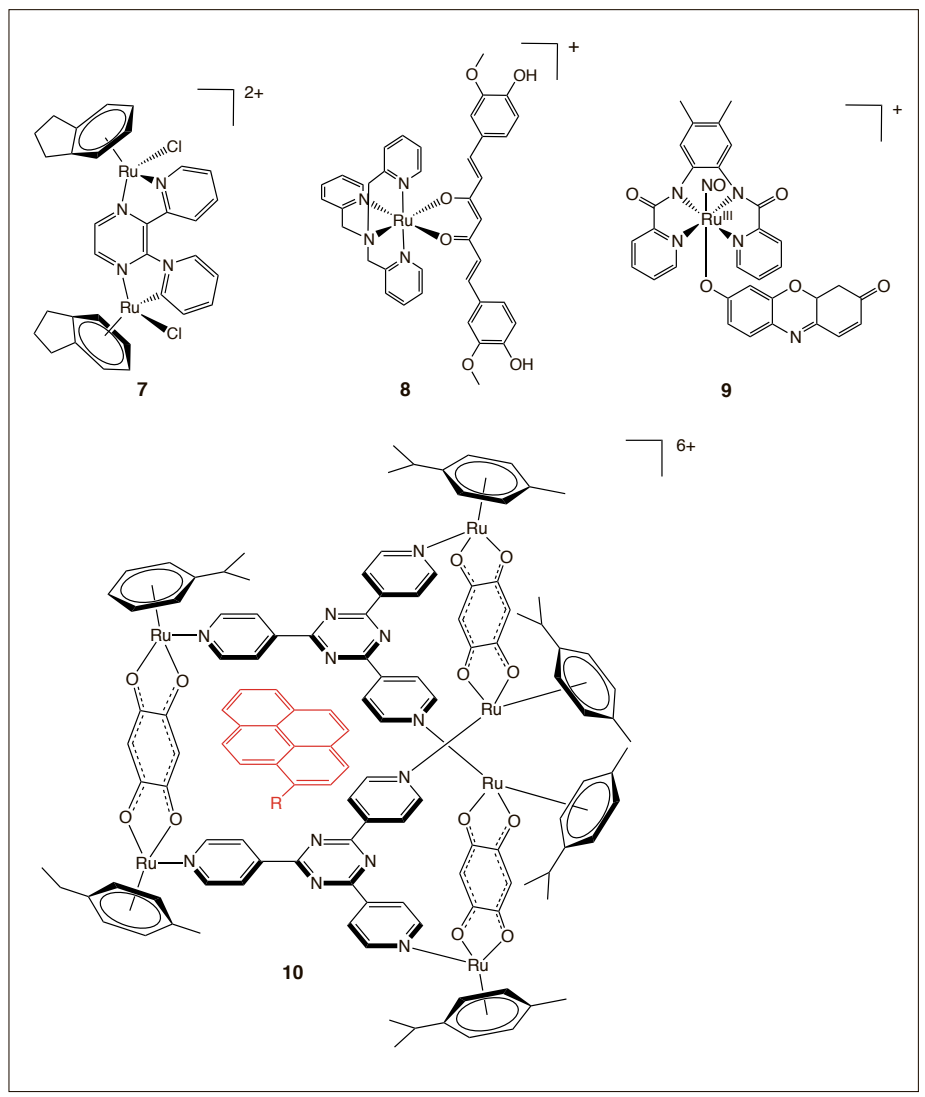

only visible up to $200 \mu \mathrm{m}$ from the spheroid edge, in the oxygenated, proliferating region. In contrast, the ruthenium complex was found to accumulate in the spheroid centre only. As the fluorescence emission of the ruthenium treated sample was of much lower intensity than that of the curcumintreated sample, it was unclear whether the

emission was due to low concentrations of free curcumin or high concentrations of coordinated curcumin. To distinguish between these two possibilities, fluorescence lifetime imaging was applied. While the lifetime of curcumin in cell free media was too short to be detected (previously reported as $(\tau 1=12 \mathrm{ps}$ and $\tau 2$
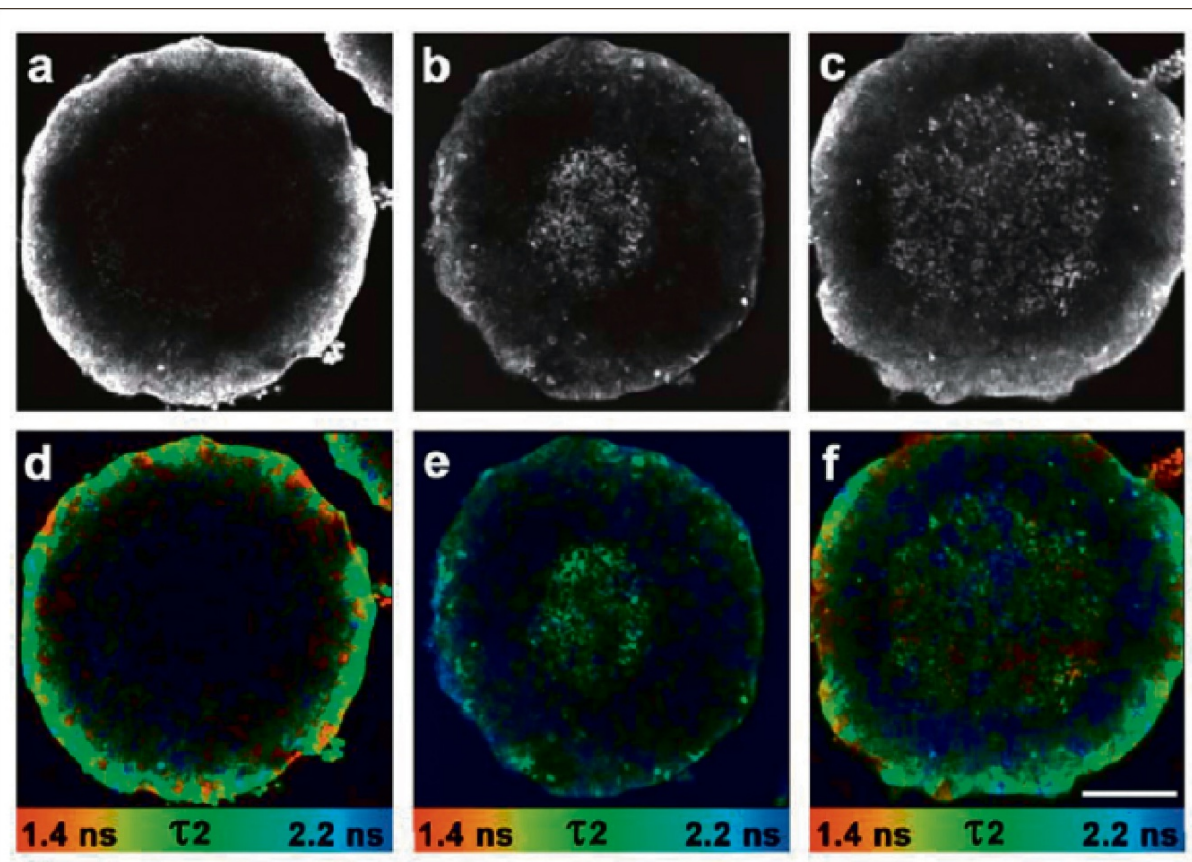

Fig. 6. Confocal fluorescence (top) and false colour fluorescence lifetime images of curcumin (left), 8 (centre) and the analogous cobalt(II) complex (right) in DLD-1 multicellular spheroids (scale bar $=100 \mu \mathrm{m})$. Reproduced from ref. [36], Chem. Sci. 2013, 4, 3731 .
$=70 \mathrm{ps}), \mathbf{8}$ had a two component decay of 353 and 2598 ps. In the treated spheroid samples, a marked difference was evident between the fluorescence lifetime of the curcumin sample $(\tau 2=1675 \mathrm{ps})$ and the ruthenium-treated sample $(\tau 2=2124 \mathrm{ps})$ indicating that the emission observed from the complex was not due to free ruthenium.

A series of ruthenium(II)arene(pta) complexes with bidentate curcumin or bisdemethoxycurcumin ligands and $p$-cymene or hexamethylbenzene arene ligands have also been investigated, where the nature of the arene ligand had a marked effect on the emission of the complex. ${ }^{[37]}$ $\left[\mathrm{Ru}\left(p\right.\right.$-cymene)curcumin (pta)] $\left(\mathrm{SO}_{3} \mathrm{CF}_{3}\right)$ and $\mathrm{Ru}(p$-cymene)desmethoxycurcumin(pta) $]\left(\mathrm{SO}_{3} \mathrm{CF}_{3}\right)$ are moderately emissive in aqueous solution while $[\mathrm{Ru}(\mathrm{hmb})$ curcumin(pta) $]\left(\mathrm{SO}_{3} \mathrm{CF}_{3}\right)$ and $[\mathrm{Ru}(\mathrm{hmb})$ desmethoxycurcumin(pta)] $\left(\mathrm{SO}_{3} \mathrm{CF}_{3}\right)$ are almost completely quenched. No confocal fluorescence microscopy studies were conducted.

Mascharak and coworkers have used fluorescent ligands to signal the lighttriggered release of a nitric oxide ligand from a ruthenium(II) carrier complex. ${ }^{[38]}$ [(Me ${ }_{2}$ ppb $\left.) \mathrm{Ru}(\mathrm{NO})(\mathrm{Resf})\right] \quad\left(\mathrm{Me}_{2} \mathrm{bpb}=\right.$ $\mathrm{N}, \mathrm{N}^{\prime}$-bis(pyridine-2-carboxamido)-1,2diamino3,4-dimethylbenzene) has a fluorescent Resorufin ligand trans to the $\mathrm{NO}(\mathbf{9}$, Fig. 5). The complex is fluorescent in the dark but release of $\mathrm{NO}$ is concurrent with oxidation of the ruthenium centre to $\mathrm{Ru}(\mathrm{III})$, which quenches the fluorescence of the Resorufin ligand. Confocal fluorescence in the dark reveals accumulation of the complex in the cytoplasm and nucleus (Fig. 7). To confirm that the Resorufin ligand remained coordinated to ruthenium, the authors also imaged cells treated with Resorufin alone, which showed nonspecific, extracellular staining. Exposure to visible light for one minute leads to a significant decrease in emission intensity. A subsequent approach uses a fluorescein ethyl ester ligand for a turn-on fluorescence response to NO release. ${ }^{[39]}$ The fluorescein ligand is quenched when coordinated to $\mathrm{Ru}(\mathrm{II})$. In aqueous solution, irradiation with $500 \mathrm{~nm}$ light induces release of NO and oxidation to $\mathrm{Ru}(\mathrm{III})$, followed by loss of the fluorescent ligand via protonation and a return of fluorescein emission. No studies in cells have been carried out.

Our work has focused on using light to release small molecule drugs from a ruthenium complex. One example, $\left[\mathrm{Ru}(\text { phen })_{2}(\text { econazole })_{2}\right]^{2+}$, is luminescent in the dark, but continued irradiation with green light induces release of one econazole ligand to generate a non-emissive aqua complex. ${ }^{[40]}$ In the dark, the complex was found to accumulate in the endoplasmic reticulum. When the cells are irradiated with light, a steady decrease in emission 

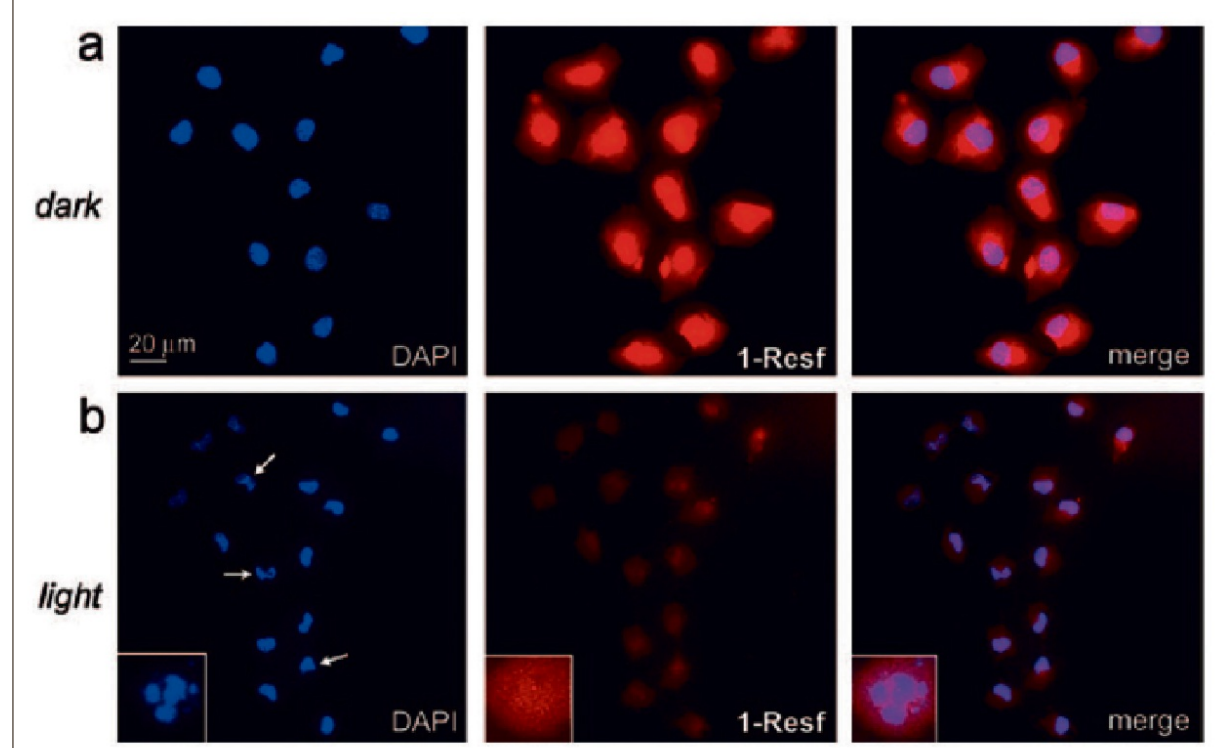

Fig. 7. MDA-MB-231 cells were loaded with $200 \mu \mathrm{M}$ solution of 9 in PBS-Ca/Mg for $1 \mathrm{~h}$ and either (a) kept in the dark or (b) exposed to 1 min visible light ( $\lambda 465 \mathrm{~nm}, 0.3 \mathrm{~W}$ measured intensity); note the nuclear degradation (arrows). Bottom insets: magnified views of a cell undergoing apoptosis. Reprinted with permission from ref. [38], J. Am. Chem. Soc. 2008, 130, 8834-8846. Copyright (2008) American Chemical Society.

is observed with increasing irradiation time, concurrent with release of the active ligand.

In an alternative approach to drug delivery, Therrien and coworkers have developed hexanuclear arene ruthenium cages to encapsulate and release a biologically active cargo (10, Fig. 5). A proof-of-concept study showed that the fluorescence of a pyrene derivative is completely quenched when encapsulated within the ruthenium cage. ${ }^{[41]}$ Destruction of the cage under basic conditions leads to a complete return in fluorescence emission. Confocal fluorescence microscopy of cells treated with the cage show increasing fluorescence emission in cytoplasmic organelles with increasing incubation time, suggesting that the fluorophore is being transported into the cells and released from the cage. Notably the emission intensity of cells treated with the pyrene alone is much lower, demonstrating the ability of the cage to increase cellular uptake.

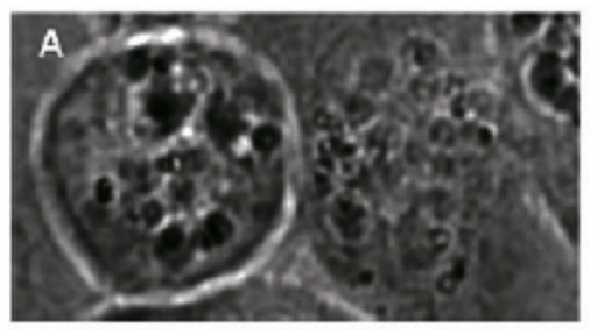

Fig. 8. Fluorescence microscopy of HeLa cells incubated with the porphyrin-encapsulated octanuclear ruthenium metalla-prism: (A) white light and (B) fluorescence. Reprinted with permission from ref. [42], J. Am. Chem. Soc. 2012, 134, 754-757. Copyright (2011) American Chemical Society.

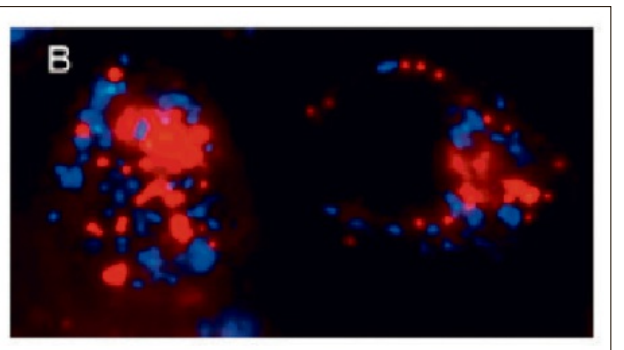

A subsequent study demonstrated the use of hexa- and octanuclear arene ruthenium cages for the transport of a porphyrin photosensitiser (Fig. 8). ${ }^{[42]}$ In this example, both the free porphyrin and the empty ruthenium cage are fluorescent, with emission maxima in the blue and red regions, respectively. However, encapsulation of the porphyrin leads to quenching of both fluorescent moieties. Significantly, the ability of the porphyrin to produce ROS is also quenched in this encapsulated form, giving a safe means of delivering the photosensitiser without the risk of systemic phototoxicity. Fluorescence microscopy of cells treated with the caged porphyrin revealed distinct regions of red and blue fluorescence in cytoplasmic organelles, indicating that the porphyrin has been released from the cage and accumulates in a different cellular region to the empty cage. This coincides with lightinduced ROS production and phototoxicity, consistent with the release of the active porphyrin from the cage. and coordination environments may allow the real-time detection of many metalbased drugs without the need to alter their structure.

\section{Fluorescence Microscopy Approaches to Analyse Metabolites after Cellular Uptake}

To eliminate the influence of a fluorescent tag on the structure and lipophilicity of a complex, an alternative approach is to probe or tag the complex with a fluorescent moiety after it has been taken up by the cell. A novel strategy by Ang and coworkers ${ }^{[43]}$ uses an RAPTA complex with an acetalfunctionalised arene ligand as a handle for conjugation and labelling. The acetal moiety is converted to an aldehyde under acidic conditions, which can then be conjugated to a hydroxylamine functionality through oxime coupling. This approach was validated in HEK cells, which were treated with the RAPTA complex then fixed with cold ethanol, activated with aqueous $\mathrm{HCl}$, then labelled with the fluorescent dye Alexa488 hydroxylamine. Cells treated with the fluorophore and RAPTA complex, showed a 20-fold increase in fluorescence with respect to cells treated with the complex alone and 7-fold increase with respect to cells treated with the fluorophore alone. While this suggests some nonspecific labelling of endogenous carbonyls within the cell, this significant increase in fluorescence validates this as a promising approach to tag complexes for pull-down assays so that specific targets could be isolated and identified.

\section{Fluorescent Probes to Detect Metal lons}

Recent years have seen an explosion in the development of fluorescent probes to sense endogenous and toxic metal ions in cellular systems. Both reaction-based and reversible probes have been explored, with the latter having the advantage of giving dynamic measurements over a certain time period. More recently, this approach has been extended to fluorescent probes for metal-based drugs and their metabolites. Ang and coworkers recently reported a reversible probe for cisplatin, which could be used for live cell imaging. ${ }^{[4]}$ This probe could detect reduction of $\mathrm{Pt}$ (IV) to $\mathrm{Pt}(\mathrm{II})$ in real time. New and coworkers have pursued a similar approach with a reversible probe that selectively senses monofunctional platinum, e.g. platinum with one labile ligand. ${ }^{[5]}$ The complementarity of the two probes allows different platinum species to be detected, potentially allowing events such as aquation and DNA binding to be monitored in real time. While this approach has only been applied to platinum-based drugs so far, the potential to adapt fluorescent probes to sense specific metal ions 


\section{Conclusions}

Recent advances in imaging techniques have allowed a deeper understanding of the behaviour of many ruthenium-based drugs, in addition to facilitating the development of several novel complexes. Despite considerable progress in this area, the techniques available for imaging metal ions in cellular systems still require a compromise between sensitivity, spatial resolution, and the ability to image live cells. With these considerations, the most effective approaches may be combining multiple modes of imaging, for example combinations of fluorescence microscopy with Nano-SIMS ${ }^{[16]}$ or SR-XRF. ${ }^{[46]}$ Many of these techniques not only map the distribution of the metal of complex, but can also give information on speciation and ligand exchange. Nano-SIMS maps the distribution of both a metal and an isotopically or iodine-labelled ligand can be detected by SR-XRF. Fluorescence microscopy can also be used to signal ligand release through an increase or decrease in emission intensity, or a change in fluorescence lifetime. The valuable insights gained from such studies will likely lead to imaging techniques playing an increasingly important role in metallodrug development.

Received: January 31, 2017

[1] a) T. Gianferrara, I. Bratsos, E. Alessio, Dalton Trans. 2009, 7588; b) C. S. Allardyce, P. J. Dyson, Dalton Trans. 2016, 45, 3201.

[2] a) A. V. Levina, A. Mitra, P. A. Lay, Metallomics 2009, 1, 458; b) C. Mari, V. Pierroz, S. Ferrari, G. Gasser, Chem. Sci. 2015, 6, 2660.

[3] L. E. Wedlock, S. J. Berners-Price, Aust. J. Chem. 2011, 64, 692.

[4] D. J. Hare, E. J. New, M. D. de Jonge, G McColl, Chem. Soc. Rev. 2015, 44, 5941

[5] a) P. Ilinski, B. Lai, Z. Cai, W. Yun, D. Legnini, T. Talarico, M. Cholewa, L. K. Webster, G. B. Deacon, S. Rainone, D. R. Phillips, A. P. J. Stampfl, Cancer Res. 2003, 63, 1776; b) M. D. Hall, R. A. Alderden, M. Zhang, P. J. Beale, Z. Cai, B. Lai, A. P. J. Stampfl, T. W. Hambley, J. Struct. Biol. 2006, 155, 38

[6] A. A. Hummer, A. Rompel, Metallomics $\mathbf{2 0 1 3}$, $5,597$.

[7] S. Vogt, B. Lai, L. Finney, B. Palmer, L. Wu, H. Harris, T. Paunesku, M. de Jonge, D. Legnini, J. Maser, P. Lay, G. Woloschak, Microscopy and Microanalytics 2007, 13, 40; see also ref. [4]
[8] J. B. Aitken, S. Antony, C. M. Weekley, B. Lai, L. Spiccia, H. H. Harris, Metallomics 2012, 4 , 1051

[9] S. Antony, J. B. Aitken, S. Vogt, B. Lai, T. Brown, L. Spiccia, H. H. Harris, J. Biol. Inorg. Chem. 2013, 7, 845 .

[10] A. A. Hummer, P. Heffeter, W. Berger, M Filipits, D. Batchelor, G. Büchel, M. A. Jakupec, B. K. Keppler, A. Rompel, J. Med. Chem. 2013, 56,1182

[11] a) R. McRae, P. Bagchi, S. Sumalekshmy, C. J. Fahrni, Chem. Rev. 2009, 109, 4780; b) C. Giesen, H. A. O. Wang, D. Schapiro, N. Zivanovic, A. Jacobs, B. Hattendorf, P. J. Schüffler, D. Grolimund, J. M. Buhmann, S. Brandt, Z. Varga, P. J. Wild, D. Günther. B. Bodenmiller, Nat. Methods 2014, 11, 417.

[12] A. E. Egger, S. Theiner, C. Kornauth, P. Heffeter, W. Berger, B. K. Keppler, C. G. Hartinger, Metallomics 2014, 6, 1616

[13] G. K. Gransbury, P. Kappen, C. J. Glover, J. N. Hughes, A. Levina, P. A. Lay, I. F. Musgrave, H. H. Harris, Metallomics, 2016, 8, 762.

[14] J. Z. Zhang, N. S. Bryce, R. Siegele, E. A. Carter, D. Paterson, M. D. d. Jonge, D. L. Howard, C. G. Ryan, T. W. Hambley, Integr. Biol. 2012, 4, 1072 .

[15] J.-L. Guerquin-Kern, T.-D. Wu, C. Quintana, A. Croisy, Biochim. Biophys. Acta, Gen. Subj. 2005, 1724, 228

[16] a) A. A. Legin, A. Schintlmeister, M. A. Jakupec, M. Galanski, I. Lichtscheidl, M. Wagner, B. K. Keppler, Chem. Sci. 2014, 5, 3135; b) L. E. Wedlock, M. R. Kilburn, R. Liu, J. A. Shaw, S. J. Berners-Price, N. P. Farrell, Chem. Commun. 2013, 49, 6944

[17] L. E. Wedlock, M. R. Kilburn, J. B. Cliff, L. Filgueira, M. Saunders, S. J. Berners-Price, Metallomics 2011, 3, 917.

[18] R F. S. Lee, S. Escrig, M. Croisier. S. ClercRosset, G. W. Knott, A. Meibom, C. A. Davey, K. Johnsson, P. J. Dyson, Chem. Commun. 2015, 51, 16486.

[19] D.-L. Ma, H.-Z. He, K.-H. Leung, D. S.-H. Chan, C.-H. Leung, Angew. Chem. Int. Ed. 2013, 52, 7666

[20] a) V, Fernández-Moreira, F. L. ThorpGreenwood, M. P. Coogan, Chem. Commun. 2010, 46, 186; b) K. K.-W. Lo, A. W.-T. Choi, W. H.-T. Law, Dalton Trans. 2012, 41, 6021.

[21] A. E. Friedman, J. C. Chambron, J. P. Sauvage, N. J. Turro, J. K. Barton, J. Am. Chem. Soc. 1990, 112, 4960

[22] V. Pierroz, T. Joshi, A. Leonidova, C. Mari, J. Schur, I. Ott, L. Spiccia, S. Ferrari, G. Gasser, J. Am. Chem. Soc. 2012, 134, 20376.

[23] a) C. Tan, S. Lai, S. Wu, S. Hu, L. Zhou, Y. Chen, M. Wang, Y. Zhu, W. Lian, W. Peng, L. Ji, A. Xu, J. Med. Chem. 2010, 53, 7613; b) C. Tan, S. Wu, S. Lai, M. Wang, Y. Chen, L. Zhou, Y. Zhu, W. Lian, W. Peng, L. Ji, A. Xu, Dalton Trans. 2011, 40, 8611 .

[24] F. Li, E. J. Harry, A. L. Bottomley, M. D. Edstein, G. W. Birrell, C. E. Woodward, F. R. Keene, J. G. Collins, Chem. Sci. 2014, 5, 685.

[25] G. Shi, S. Monro, R. Hennigar, J. Colpitts, J. Fong, K. Kasimova, H. Yin, R. DeCoste, C. Spencer, L. Chamberlain, A. Mandel, L. Lilge,
S. A. McFarland, Coord. Chem. Rev. 2015, 282, 127.

[26] C. Mari, V. Pierroz, R. Rubbiani, M. Patra, J. Hess, B. Spingler, L. Oehninger, J. Schur, I. Ott, L. Salassa, S. Ferrari, G. Gasser, Chem. Eur. J. 2014, 20, 14421

[27] M. Dickerson, Y. Sun, B. Howerton, E. C. Glazer, Inorg. Chem. 2014, 53, 10370.

[28] F. Schmitt, P. Govindaswamy, O. Zava, G. SüssFink, L. Juillerat-Jeanneret, B. Therrien, J. Biol. Inorg. Chem. 2009, 14, 101.

[29] A. Kurzwernhart, W. Kandioller, C. Bartel, S. Bachler, R. Trondl, G.Muhlgassner, M. A. Jakupec, V. B. Arion, D. Marko, B. K. Keppler, C. G. Hartinger, Chem. Commun. 2012, 48 , 4839.

[30] Q. Zhou, W. Lei, Y. Chen, C. Li, Y. Hou, B Zhang, X. Wang, Chem. Eur. J. 2012, 18, 8617.

[31] C. A. Vock, W. H. Ang, C. Scolaro, A. D Phillips, L. Lagopoulos, L. Juillerat-Jeanneret, G. Sava, R. Scopelliti, P. J. Dyson, J. Med. Chem. 2007, 50, 2166.

[32] A. A. Nazarov, J. Risse, W. H. Ang, F. Schmitt, O.Zava, A. Ruggi, M. Groessl, R. Scopelitti, L. Juillerat-Jeanneret, C. G. Hartinger, P. J. Dyson, Inorg. Chem. 2012, 51, 3633.

[33] K. J. Kilpin, C. M. Clavel, F. Edafe, P. J. Dyson, Organometallics 2012, 31, 7031.

[34] S. Antony, J. C. Morris, T. D. M. Bell, T. Brown, L. Spiccia, H. H. Harris, Aust. J. Chem. 2014, $67,1711$.

[35] S. W. Magennis, A. Habtemariam, O. Novakova J. B. Henry, S. Meier, S. Parsons, I. D. H. Oswald, V. Brabec, P. J. Sadler, Inorg. Chem. 2007, 46, 5059.

[36] A. K. Renfrew, N. S. Bryce, T. W. Hambley, Chem. Sci. 2013, 4, 3731.

[37] R. Pettinari, F. Marchetti, F. Condello, C. Pettinari, G. Lupidi, R. Scopelliti, S. Mukhopadhyay, T. Riedel, P. J. Dyson, Organometallics 2014, 33, 3709.

[38] M. J. Rose, N. L. Fry, R. Marlow, L. Hinck, P. K. Mascharak, J. Am. Chem. Soc. 2008, 130, 8834.

[39] N. L. Fry, J. Wei, P. K. Mascharak, Inorg. Chem. 2011, 50, 9045.

[40] N. Karaoun, A. K. Renfrew, Chem. Commun $\mathbf{2 0 1 5}, 51,14038$

[41] O. Zava, J. Mattsson, B. Therrien, P. J. Dyson, Chem. Eur. J. 2011, 17, 9669.

[42] F. Schmitt, J. Freudenreich, N. P. E. Barry, L. Juillerat-Jeanneret, G. Süss-Fink, B. Therrien, J. Am. Chem. Soc. 2012, 134, 754.

[43] Y. Q. Tan, P. J. Dyson, W. H. Ang, Organometallics 2011, 30, 5965.

[44] D. Montagner, S. Q. Yap, W. H. Ang, Angew. Chem. Int. Ed. 2013, 52, 11785.

[45] C. Shen, B. D. W. Harris, L. J. Dawson, K. A. Charles, T. W. Hambley, E. J. New, Chem Commun. 2015, 51, 6312.

[46] A. Grubman, S. A. James, J. James, C. Duncan, I. Volitakis, J. L. Hickey, P. J. Crouch, P. S. Donnelly, K. Kanninen, J. R. Liddell, S. L. Cotman, M. D. de Jonge, A. R. White, Chem. Sci. 2014, 5, 2503. 